\title{
A New Truncated Muth Generated Family of Distributions with Applications
}

\author{
Abdullah M. Almarashi, ${ }^{1}$ Farrukh Jamal $\left(\mathbb{D},{ }^{2}\right.$ Christophe Chesneau (iD, ${ }^{3}$ \\ and Mohammed Elgarhy (iD) \\ ${ }^{1}$ Statistics Department, Faculty of Science, King AbdulAziz University, Jeddah, Saudi Arabia \\ ${ }^{2}$ Department of Statistics, The Islamia University of Bahawalpur, Bahawalpur, Punjab 63100, Pakistan \\ ${ }^{3}$ Université de Caen, LMNO, Campus II, Science 3, Caen 14032, France \\ ${ }^{4}$ The Higher Institute of Commercial Sciences, Al Mahalla Al Kubra, Algarbia 31951, Egypt \\ Correspondence should be addressed to Mohammed Elgarhy; m_elgarhy85@sva.edu.eg
}

Received 24 July 2021; Accepted 7 September 2021; Published 21 September 2021

Academic Editor: Yi Su

Copyright (c) 2021 Abdullah M. Almarashi et al. This is an open access article distributed under the Creative Commons Attribution License, which permits unrestricted use, distribution, and reproduction in any medium, provided the original work is properly cited.

\begin{abstract}
In recent years, the Muth distribution has been used for the construction of accurate statistical models, with applications in various applied fields. In this paper, we use a truncated-composed scheme to create a new unit Muth distribution, from which we motivate a more general family of continuous distributions called the truncated Muth generated family. The key benefits of this family are its analytical simplicity, connections with the exponential generated family, and flexibility conferred on any parental distribution. In particular, it improves the capability of the functions of the parental distribution, enhancing their peak, asymmetry, tail, and flatness levels, among others. The characteristics of quantile and moment measures and functions of the truncated Muth generated family are described in detail. As a concrete example, a particular distribution that extends the Weibull distribution is highlighted. In an applied part, the parameters are calculated using the maximum likelihood procedure. We use a comprehensive simulation analysis to demonstrate the accuracy of the derived estimates. The revised Weibull model is then used to fit two real-world datasets. The new model is shown to be more suited to these datasets than other competing models.
\end{abstract}

\section{Introduction}

The Muth (M) distribution, in its most basic form, is a lifetime distribution introduced by [1]. It is mathematically defined by the following one-parameter cumulative distribution function (cdf):

$$
F_{M}(x)=1-\exp \left[\alpha x-\frac{1}{\alpha}\left(e^{\alpha x}-1\right)\right], \quad x \geq 0,
$$

with $\alpha \in(0,1]$, and $F_{M}(x)=0$ for $x<0$. The following properties are satisfied. As $\alpha$ approaches 0 , the $M$ distribution transforms into a classical exponential distribution with the parameter equal to one. Its right tail is less weighted than that of some other lifetime distributions, and it has enough versatility to suit a large panel of lifetime data sets properly, especially those resulting from reliability experiments. Many of these properties are developed in $[1-3]$. The $M$ distribution was later generalized by Jodra et al. [4] using the power transform. It is also possible to refer to the exponentiated Tessier distribution by Sharma et al. [5], where it provides an in-depth analytical connection with the exponentiated version of the $M$ distribution.

Also, an innovative approach has sought to develop generic families of continuous distributions that generalize or expand a parental distribution using specific mathematical schemes. By combining the type II of the T-X scheme by Alzaatreh et al. [6] and the $M$ distribution, in [7], the authors proposed the $M$ generated $(M-G)$ class of distributions with the perspective of creating attractive distributions for statisticians. Concretely, the $M-G$ class is defined by the following cdf: 


$$
F_{M-G}(x)=G(x)^{-\alpha} \exp \left\{-\frac{1}{\alpha}\left[G(x)^{-\alpha}-1\right]\right\}, \quad x \in \mathbb{R}
$$

where $G(x)$ refers to the cdf of a continuous distribution, that we call the parental distribution. Then, to demonstrate the importance of the $M-G$ class, in [7], the authors looked at the $M$ uniform, $M$ Rayleigh, $M$ Lomax, $M$ exponential, and $M$ Weibull distributions. The graphs show that the main functions, and the probability density functions (pdfs) and hazard rate functions (hrfs) in particular, have a variety of curvatures, demonstrating their ability to model heterogeneous phenomena. This is shown in [7] using the $M$ Weibull distribution as a parangon of the $M-G$ class and some data by Abouelmagd et al. [8] on aircraft windshield failure times. In fact, it is shown that the $M$ Weibull model adjusts these data better than other extensions of the Weibull models. An extension of the $M-G$ family was proposed by [9] through the transmuted scheme. As an important remark made in [9], one can take $\alpha$ such that $\alpha \in(\infty, 1] /\{0\}$, a condition that significantly improves the modeling ability of the $M-G$ family, among others. This condition on $\alpha$ will be used throughout the rest of the study. To conclude this paragraph, we mention that another type of $M-G$ family has been established in [10], also based on the $T-X$ transformation.

In this paper, we contribute to the capability of the $M$ distribution to generate flexible distributions by considering the truncated-composed scheme. A list of recent families of distributions based on this scheme is the following: truncated Fréchet generated (TFG) family by Abid and Abdulrazak [11], truncated Weibull generated (TWG) family by Najarzadegan et al. [12], truncated inverted Kumaraswamy generated (TIKG) family by Bantan et al. [13], type II truncated Fréchet generated (TIITFG) family by Aldahlan [14], truncated Cauchy power generated (TCPG) family by Aldahlan et al. [15], exponentiated truncated inverse Weibull generated (ETIWG) family by Almarashi et al. [16], truncated Burr generated (TBG) family by Jamal et al. [17], truncated generalized Fréchet generated (TGFG) family by ZeinEldin et al. [18], truncated inverse Lomax generated (TILG) family by Algarni et al. [19], and truncated Burr X generated (TBX) family by Bantan et al. [20]. Despite this extensive literature, there is no work on what can be called the new truncated $M$ generated (NTM-G) distribution. The main interest of this family is to provide a simplified alternative to the $M-G$ family, while using the functionalities of the $M$ distribution to extend the modeling features of any parental distribution.

The following plan is used to illustrate this claim. Section 2 is devoted to a special truncated $M$ distribution belonging to the family of unit distributions. It is new and also is the key ingredient in defining our main general family. We will look at some of its most interesting properties, with a focus on quantitative analysis and moment analysis. The considered TM-G family is defined in Section 3. After the investigation of its related functions, we perform a quantile analysis, followed by a moment analysis. In addition, a numerical work is given on some moment measures. Section 4 is the applied part; we show concretely how the TM-G family can be used to estimate a distribution from data. The maximum likelihood (ML) procedure is employed, and reinforced by a simulation study to guarantee the effectiveness of the method. Real data are then analysed through comparable models, showing that our model is competitive and more accurate than some models derived from the $M-G$ family. A conclusion is given in Section 5 .

\section{The Unit Truncated M Distribution}

2.1. Motivation. The creation of unit interval distributions is growing rapidly in the literature. As the main explanation, these distributions are useful for modeling proportions, percentages, and rates that are defined at a unit interval. Modern applications are numerous in the fields of psychology, economics, biology, and engineering. For more information on this topic, see [21-24] as well as the references therein. This section is dedicated to the unit truncated $M$ distribution, which will be the main ingredient to the NTM-G family. It is worth noting that this unit distribution is not documented in the literature to our knowledge.

2.2. Presentation. Based on the cdf of the $M$ distribution as described in equation (1), we propose a new unit distribution, called the new truncated $M$ (NTM) distribution. It is defined by the following cdf:

$$
F_{N T M}(x)=\frac{F_{M}(x)}{F_{M}(1)}, \quad x \in(0,1),
$$

and the following adjustments at the boundaries: $F_{N T M}(x)=0$ for $x<0$ and $F_{N T M}(x)=1$ for $x>1$, that is, for $x \in(0,1)$, we have

$$
F_{N T M}(x)=C_{\alpha}\left\{1-\exp \left[\alpha x-\frac{1}{\alpha}\left(e^{\alpha x}-1\right)\right]\right\},
$$

with $C_{\alpha}=\left\{1-\exp \left[\alpha-\left(e^{\alpha}-1\right) / \alpha\right]\right\}^{-1}$. We recall that $\alpha \in(-\infty, 1] /\{0\}$. To our knowledge, there is no reference to the NTM distribution in the literature. The NTM distribution, like the other unit distributions in the abovementioned references, can be used to interpret any proportional-like data. As a result, it offers an alternative to some useful one-parameter unit distributions, such as the power, beta, and the Topp-Leone distributions by Topp and Leone [25].

As a basic property, when $\alpha$ decreases to 0 , the NTM distribution has a unit truncated exponential distribution as the limit distribution.

In complement to the cdf, note that the pdf of the NTM distribution is specified by

$$
f_{N T M}(x)=C_{\alpha}\left(e^{\alpha x}-\alpha\right) \exp \left[\alpha x-\frac{1}{\alpha}\left(e^{\alpha x}-1\right)\right], \quad x \in(0,1),
$$

with $f_{N T M}(x)=0$ for $x<0$ and $f_{N T M}(x)=1$ for $x>1$, and the hrf is obtained as

$$
h_{N T M}(x)=\frac{C_{\alpha}\left(e^{\alpha x}-\alpha\right) \exp \left[\alpha x-\left(e^{\alpha x}-1\right) / \alpha\right]}{1-C_{\alpha}\left\{1-\exp \left[\alpha x-\left(e^{\alpha x}-1\right) / \alpha\right]\right\}}, \quad x \in(0,1) \text {, }
$$


with $h_{N T M}(x)=0$ for $x<0$ and $h_{N T M}(x)=1$ for $x>1$. The shape behavior of these two functions reveals a flexible model that may be useful for a variety of statistical applications. We, however, omit the details of this aspect and prefer to focus on the quantile analysis and moment analysis of the NTM distribution, which will have important uses for the coming NMTG family.

2.3. Quantile Analysis. The quantile function (qf) of the NTM distribution is described in the following result. As for the classical $M$ distribution, the Lambert function plays a central role.

Proposition 1. The of of the NTM distribution is

$$
Q_{N T M}(u)=\frac{1}{\alpha} \log \left[-\alpha W\left(\frac{u d_{\alpha}-1}{\alpha e^{1 / \alpha}}\right)\right], \quad u \in(0,1),
$$

where $d_{\alpha}=C_{\alpha}^{-1}=1-\exp \left[\alpha-\left(e^{\alpha}-1\right) / \alpha\right]$ and $W(x)$ is the Lambert function.

Proof. The following equation, $F_{N T M}(x)=u$ with respect to $x$, is used to determine the qf. This equation is equivalent to

$$
\exp \left[\alpha x-\frac{1}{\alpha}\left(e^{\alpha x}-1\right)\right]=1-u d_{\alpha}
$$

and by applying (Corollary 2 in [2]) with " $u d_{\alpha}$ " instead of " $u$," we get the stated result.

The concept of qf has been used in a variety of publications, both theoretical and practical. Essentially, we can use it to define the three main quartiles of a distribution; for the NTM distribution, they are given by $Q_{1}=Q_{N T M}(1 / 4)$, $Q_{2}=Q_{N T M}(1 / 2)$, and $Q_{3}=Q_{N T M}(3 / 4)$. The median of the NTM distribution is simply $Q_{2}$, that is,

$$
Q_{2}=\frac{1}{\alpha} \log \left[-\alpha W\left(\frac{d_{\alpha}-2}{2 \alpha e^{1 / \alpha}}\right)\right] .
$$

The $\mathrm{qf}$ is also the basis of some distributional measures, such as the skewness measure of Bowley expressed as

$$
B=\frac{Q_{3}-2 Q_{2}+Q_{1}}{Q_{3}-Q_{1}},
$$

and the kurtosis measure of Moors is defined by

$$
K=\frac{O_{7}-O_{5}+O_{3}-O_{1}}{Q_{3}-Q_{1}},
$$

where $O_{i}$ denotes the $i^{\text {th }}$ octile of the NTM distribution, i.e., $O_{i}=Q_{N T M}(i / 8), i=1,3,5$ and 7 . As a last remark, we can generate values from the NTM distribution by exploiting the fact that, for any random variable $U$ with the unit uniform distribution, $Q_{N T M}(U)$ has the NTM distribution. In other words, using the quantile transformation, values generated by the unit uniform distribution are converted to values created by the NTM distribution. More information on the topic of quantitative analysis can be found in the book of [26].

2.4. Moment Analysis. In this section, we perform a moment analysis. With this aim, we introduce the following special integral function:

$$
\mathfrak{\Im}(a, b, c, d, \pm)=\int_{a}^{b}( \pm y)^{c}(\log ( \pm y))^{d} e^{-y} \mathrm{~d} y,
$$

for $a, b, c$, and $d$ in such a way that this special integral exists. Connections with well-established integral functions, including gamma and general exponential integral functions, exist. We can also view this integral as a truncated version of the exponential transform of the power-log function $u(x)=$ $( \pm x)^{c}(\log ( \pm x))^{d}$.

The moment generating function (mgf) of the NTM distribution is described in the following result.

Proposition 2. Let $X$ be a random variable with the NTM distribution. The $m g f$ of $X$ is defined by $M(t)=\mathbb{E}\left(e^{t X}\right)$ with $t \in \mathbb{R}$; it always exists and can be expressed as

$$
M(t)=C_{\alpha} e^{1 / \alpha} \times \begin{cases}(-\alpha)^{t / \alpha+1}\left[\Im\left(\frac{1}{\alpha}, \frac{e^{\alpha}}{\alpha}, \frac{t}{\alpha}, 0,-\right)+\Im\left(\frac{1}{\alpha}, \frac{e^{\alpha}}{\alpha}, \frac{t}{\alpha}+1,0,-\right)\right], & \text { for } \alpha<0, \\ \alpha^{t / \alpha+1}\left[\Im\left(\frac{1}{\alpha}, \frac{e^{\alpha}}{\alpha}, \frac{t}{\alpha}+1,0,+\right)-\Im\left(\frac{1}{\alpha}, \frac{e^{\alpha}}{\alpha}, \frac{t}{\alpha}, 0,+\right)\right], & \text { for } \alpha \in(0,1],\end{cases}
$$

where $\mathfrak{I}(a, b, c, d, \pm)$ is the integral function defined in equation (12).

Proof. By changing the variable $y=e^{\alpha x} / \alpha$, we have

$$
\begin{aligned}
M(t) & =\int_{-\infty}^{+\infty} e^{t x} f_{N T M}(x) \mathrm{d} x=\int_{0}^{1} e^{t x}\left(e^{\alpha x}-\alpha\right) \exp \left[\alpha x-\frac{1}{\alpha}\left(e^{\alpha x}-1\right)\right] \mathrm{d} x \\
& =C_{\alpha} \alpha e^{1 / \alpha} \int_{1 / \alpha}^{e^{\alpha} / \alpha}(\alpha y)^{t / \alpha}(y-1) e^{-y} \mathrm{~d} y .
\end{aligned}
$$


If $\alpha<0$, we can write

$$
\begin{aligned}
M(t) & =C_{\alpha}(-\alpha)^{t / \alpha+1} e^{1 / \alpha} \int_{1 / \alpha}^{e^{\alpha} / \alpha}(-y)^{t / \alpha}(1-y) e^{-y} \mathrm{~d} y \\
& =C_{\alpha}(-\alpha)^{t / \alpha+1} e^{1 / \alpha}\left[\int_{1 / \alpha}^{e^{\alpha} / \alpha}(-y)^{t / \alpha} e^{-y} \mathrm{~d} y+\int_{1 / \alpha}^{e^{\alpha} / \alpha}(-y)^{t / \alpha+1} e^{-y} \mathrm{~d} y\right] \\
& =C_{\alpha}(-\alpha)^{t / \alpha+1} e^{1 / \alpha}\left[\Im\left(\frac{1}{\alpha}, \frac{e^{\alpha}}{\alpha}, \frac{t}{\alpha}, 0,-\right)+\Im\left(\frac{1}{\alpha}, \frac{e^{\alpha}}{\alpha}, \frac{t}{\alpha}+1,0,-\right)\right]
\end{aligned}
$$

This ends the proof of the proposition.

Remark 1. For the case $\alpha \in(0,1]$, by considering the incomplete upper gamma function specified by $\Gamma(a, b)=\int_{b}^{+\infty} y^{a-1} e^{-y} \mathrm{~d} y$ for $a>0$ and $b \geq 0$ and the following recurrence relationship $\Gamma(x+1, b)=x \Gamma$ $(x, b)+b^{x} e^{-b}$, we have

On the contrary, if $\alpha \in(0,1]$, we can write

$$
\begin{aligned}
M(t) & =C_{\alpha} \alpha^{t / \alpha+1} e^{1 / \alpha} \int_{1 / \alpha}^{e^{\alpha} / \alpha} y^{t / \alpha}(y-1) e^{-y} \mathrm{~d} y \\
& =C_{\alpha} \alpha^{t / \alpha+1} e^{1 / \alpha}\left[\int_{1 / \alpha}^{e^{\alpha} / \alpha} y^{t / \alpha+1} e^{-y} \mathrm{~d} y-\int_{1 / \alpha}^{e^{\alpha} / \alpha} y^{t / \alpha} e^{-y} \mathrm{~d} y\right] \\
& =C_{\alpha} \alpha^{t / \alpha+1} e^{1 / \alpha}\left[\Im\left(\frac{1}{\alpha}, \frac{e^{\alpha}}{\alpha}, \frac{t}{\alpha}+1,0,+\right)-\Im\left(\frac{1}{\alpha}, \frac{e^{\alpha}}{\alpha}, \frac{t}{\alpha}, 0,+\right)\right] .
\end{aligned}
$$

$$
\begin{aligned}
M(t) & =C_{\alpha} \alpha^{1+t / \alpha} e^{1 / \alpha}\left[\Gamma\left(\frac{t}{\alpha}+2, \frac{1}{\alpha}\right)-\Gamma\left(\frac{t}{\alpha}+1, \frac{1}{\alpha}\right)-\Gamma\left(\frac{t}{\alpha}+2, \frac{e^{\alpha}}{\alpha}\right)+\Gamma\left(\frac{t}{\alpha}+1, \frac{e^{\alpha}}{\alpha}\right)\right] \\
& =C_{\alpha} \alpha^{t / \alpha} e^{1 / \alpha} t\left[\Gamma\left(\frac{t}{\alpha}+1, \frac{1}{\alpha}\right)-\Gamma\left(\frac{t}{\alpha}+1, \frac{e^{\alpha}}{\alpha}\right)\right]+C_{\alpha}\left\{1-\exp \left[t+\alpha-\left(e^{\alpha}-1\right) / \alpha\right]\right\}
\end{aligned}
$$

Proposition 3. Let $s$ be a positive integer and $X$ be a random variable with the NTM distribution. Then, the sth ordinary moment of $X$ defined by $m(s)=\mathbb{E}\left(X^{s}\right)$ always exists and can be expressed as

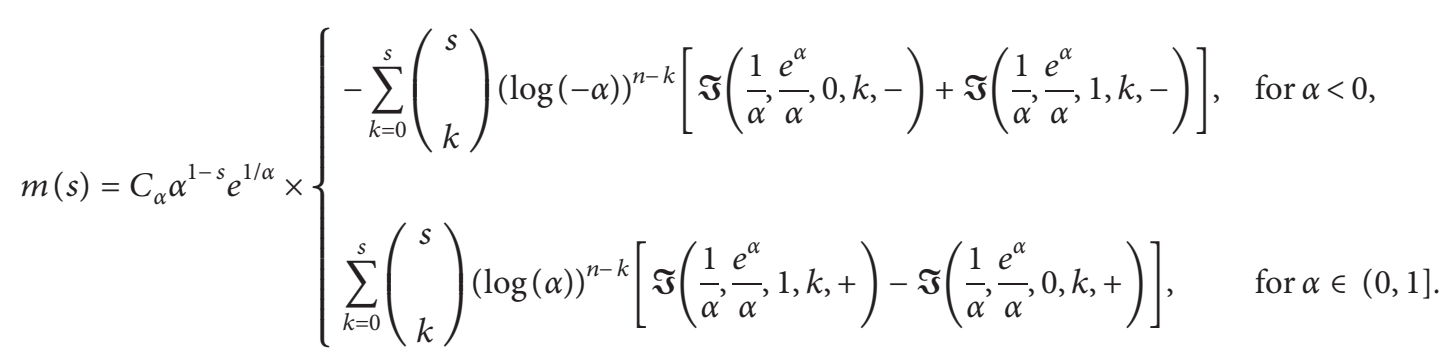

Proof. We proceed in the same way as the proof of Proposition 2. By making the change of variable $y=e^{\alpha x} / \alpha$, we have

$$
\begin{aligned}
m(s) & =\int_{-\infty}^{+\infty} x^{s} f_{N T M}(x) \mathrm{d} x \\
& =C_{\alpha} \int_{0}^{1} x^{s}\left(e^{\alpha x}-\alpha\right) \exp \left[\alpha x-\frac{1}{\alpha}\left(e^{\alpha x}-1\right)\right] \mathrm{d} x \\
& =C_{\alpha} \alpha^{1-s} e^{1 / \alpha} \int_{1 / \alpha}^{e^{\alpha} / \alpha}[\log (\alpha y)]^{s}(y-1) e^{-y} \mathrm{~d} y .
\end{aligned}
$$

If $\alpha<0$, as a result of the binomial formula, we have

$$
\begin{aligned}
m(s)= & C_{\alpha} \alpha^{1-s} e^{1 / \alpha} \int_{1 / \alpha}^{e^{\alpha} / \alpha}(\log (\alpha)+\log (-y))^{s}(y-1) e^{-y} \mathrm{~d} y \\
= & -C_{\alpha} \alpha^{1-s} e^{1 / \alpha} \sum_{k=0}^{s}\left(\begin{array}{c}
s \\
k
\end{array}\right)(\log (-\alpha))^{n-k} \\
& \int_{1 / \alpha}^{e^{\alpha} / \alpha}(\log (-y))^{k}(1-y) e^{-y} \mathrm{~d} y
\end{aligned}
$$




$$
\begin{aligned}
= & -C_{\alpha} \alpha^{1-s} e^{1 / \alpha} \sum_{k=0}^{s}\left(\begin{array}{l}
s \\
k
\end{array}\right)(\log (-\alpha))^{n-k} \\
& {\left[\Im\left(\frac{1}{\alpha}, \frac{e^{\alpha}}{\alpha}, 0, k,-\right)+\Im\left(\frac{1}{\alpha}, \frac{e^{\alpha}}{\alpha}, 1, k,-\right)\right] . }
\end{aligned}
$$

On the contrary, if $\alpha \in(0,1]$, by invoking the same arguments, we get

$$
\begin{aligned}
m(s)= & C_{\alpha} \alpha^{1-s} e^{1 / \alpha} \int_{1 / \alpha}^{e^{\alpha} / \alpha}(\log (\alpha)+\log (y))^{s}(y-1) e^{-y} \mathrm{~d} y \\
= & C_{\alpha} \alpha^{1-s} e^{1 / \alpha} \sum_{k=0}^{s}\left(\begin{array}{c}
s \\
k
\end{array}\right)(\log (\alpha))^{n-k} \\
& \int_{1 / \alpha}^{e^{\alpha} / \alpha}(\log (y))^{k}(1-y) e^{-y} \mathrm{~d} y \\
= & C_{\alpha} \alpha^{1-s} e^{1 / \alpha} \sum_{k=0}^{s}\left(\begin{array}{c}
s \\
k
\end{array}\right)(\log (\alpha))^{n-k} \\
& {\left[\Im\left(\frac{1}{\alpha}, \frac{e^{\alpha}}{\alpha}, 1, k,+\right)-\Im\left(\frac{1}{\alpha}, \frac{e^{\alpha}}{\alpha}, 0, k,+\right)\right] . }
\end{aligned}
$$

The proof is now completed.

Based on Proposition 3, the mean $m(1)$ and standard deviation $\sigma$ of $X$ can be derived. Also, by using standard relations, we can determine the sth moment of $X$ about the mean given as $m^{\dagger}(s)=\mathbb{E}\left[(X-m(1))^{s}\right]$. The moments skewness and kurtosis coefficients of $X$ are given by $S=$ $m^{\dagger}(3) / \sigma^{3}$ and $K=m^{\dagger}(4) / \sigma^{4}$.

While adjusting the settings of the parameter $\alpha$, Table 1 displays some numerical values for the measures above.

We can note from Table 1 that, for the considered values only, when $\alpha$ increases, $m(1)$ and $S$ are increased. But $K$ increases when $\alpha<0$ and decreases when $\alpha>0$. However, the rigorous monotonicity of these measures needs further investigation that we omit here.

\section{NTM-G Family}

The NTM-G family is now the focus of attention.

3.1. Presentation. The truncated-composition scheme is applied to define the NTM-G family. By considering a parental cdf denoted by $G(x)$ and the NTM distribution, we define the NTM generated (NTM-G) family by the cdf obtained by the following composition: $F_{N T M-G}(x)=F_{N T M}(G(x)), x \in \mathbb{R}$, that is,
TABLE 1: Values of moment measures of the NTM distribution for different values of $\alpha$.

\begin{tabular}{lccccccc}
\hline$\alpha$ & $m(1)$ & $m(2)$ & $m(3)$ & $m(4)$ & $\sigma^{2}$ & $S$ & $K$ \\
\hline-0.9 & 0.849 & 1.404 & 4 & 16.438 & 0.682 & 2.928 & 15.827 \\
-0.7 & 1.003 & 1.948 & 6.778 & 34.889 & 0.942 & 3.211 & 18.512 \\
-0.5 & 1.194 & 2.755 & 12.131 & 83.006 & 1.328 & 3.703 & 24.107 \\
-0.3 & 1.402 & 3.685 & 20.563 & 201.797 & 1.719 & 4.691 & 40.04 \\
-0.1 & 1.537 & 3.474 & 14.129 & 106.981 & 1.112 & 4.581 & 42.553 \\
0.05 & 1.605 & 3.064 & 8.404 & 29.536 & 0.487 & 5.65 & 12.752 \\
0.1 & 1.629 & 2.983 & 7.594 & 24.109 & 0.33 & 8.777 & 9.171 \\
\hline
\end{tabular}

$$
F_{N T M-G}(x)=C_{\alpha}\left\{1-\exp \left[\alpha G(x)-\frac{1}{\alpha}\left(e^{\alpha G(x)}-1\right)\right]\right\}, \quad x \in \mathbb{R} .
$$

The main motivations for defining this family are (i) to transpose the functionalities of the NTM distribution to increase those of the parental distribution defined by $G(x)$ and (ii) offer a more simple alternative to the former $M-G$ family introduced in [7]. In some senses, it is more connected with the true nature of the $M$ distribution.

The pdf is obtained as

$$
f_{N T M-G}(x)=C_{\alpha} g(x)\left(e^{\alpha G(x)}-\alpha\right) \exp \left[\alpha G(x)-\frac{1}{\alpha}\left(e^{\alpha G(x)}-1\right)\right], \quad x \in \mathbb{R},
$$

where $g(x)$ refers to the pdf derived to $G(x)$. The hrf is given as

$$
h_{N T M-G}(x)=\frac{C_{\alpha} g(x)\left(e^{\alpha G(x)}-\alpha\right) \exp \left[\alpha G(x)-\left(e^{\alpha G(x)}-1\right) / \alpha\right]}{1-C_{\alpha}\left\{1-\exp \left[\alpha G(x)-\left(e^{\alpha G(x)}-1\right) / \alpha\right]\right\}}, \quad x \in \mathbb{R} .
$$

These functions are modulable with respect to $\alpha$, and the definition of the parental distribution is governed by $G(x)$ and $g(x)$. We thus extend the scope of this parental distribution through the use of the NTM strategy.

3.2. An Extended Weibull Distribution. Here, we aim to extend the Weibull distribution through the NTM scheme. To begin, the Weibull distribution with parameters $c>0$ and $k>0$ is specified by the following cdf and pdf:

$$
G_{W}(x)=1-e^{-(x / c)^{k}}, \quad x>0,
$$

with $G_{W}(x)=0$ for $x \leq 0$, and

$$
g_{W}(x)=\frac{k}{c}\left(\frac{x}{c}\right)^{k-1} e^{-(x / c)^{k}}, \quad x>0,
$$

with $g_{W}(x)=0$ for $x \leq 0$, respectively. By substituting these functions for those defining the NTM-G family, we introduce the NTMW distribution with the following cdf and pdf:

$$
F_{N T M W}(x)=C_{\alpha}\left\{1-\exp \left[\alpha\left(1-e^{-(x / c)^{k}}\right)-\frac{1}{\alpha}\left(e^{\alpha\left(1-e^{-\left(x / c c^{k}\right.}\right)}-1\right)\right]\right\}, \quad x>0,
$$


and $F_{N T M W}(x)=0$ for $x<0$, and

$$
\begin{aligned}
f_{N T M W}(x)= & C_{\alpha} \frac{k}{c}\left(\frac{x}{c}\right)^{k-1} e^{-(x / c)^{k}}\left(e^{\alpha\left(1-e^{-(x / c)^{k}}\right)}-\alpha\right) \exp \\
& {\left[\alpha\left(1-e^{-(x / c)^{k}}\right)-\frac{1}{\alpha}\left(e^{\alpha\left(1-e^{-(x / c)^{k}}\right)}-1\right)\right], \quad x>0, }
\end{aligned}
$$

and $f_{N T M W}(x)=0$ for $x \leq 0$, respectively.

Also, the hrf of the NTMW distribution is given by

$$
h_{N T M W}(x)=\frac{C_{\alpha}(k / c)(x / c)^{k-1} e^{-(x / c)^{k}}\left(e^{\alpha\left(1-e^{-(x / c)^{k}}\right)}-\alpha\right) \exp \left[\alpha\left(1-e^{-(x / c)^{k}}\right)-\left(e^{\alpha\left(1-e^{-(x / c)^{k}}\right)}-1\right) / \alpha\right]}{1-C_{\alpha}\left\{1-\exp \left[\alpha\left(1-e^{-(x / c)^{k}}\right)-\left(e^{\alpha\left(1-e^{-(x / c)^{k}}\right)}-1\right) / \alpha\right]\right\}}, \quad x>0,
$$

and $h_{N T M W}(x)=0$ for $x \leq 0$. Thus, the NTMW distribution offers a new three-parameter distribution that modifies the analytical structure of the Weibull distribution by adding more tuning parameters. As a visual approach, Figure 1 depicts some plots showing different shapes of the pdf and hrf of the NTMW distribution.

From Figure 1, we observe that the pdf of the NTMW distribution has monotonic and nonmonotonic shapes, including diverse decreasing and unimodal shapes, with left and right skewed characteristics. The shape panel of the hrf is quite extensive; we see increasing (concave or convex), decreasing, almost constant, and upside-down shapes. All of these observations demonstrate the flexibility of the NTMW model, which justifies its use in applied statistical scenarios. The pdf and hrf of the MW distribution display in [7] do not have as many shapes. The possible applications of this model will be found in the concrete examples in the applied section of the paper.

3.3. Quantile Analysis. The quantile features of the NTM-G family can be easily obtained using the quantile analysis of the NTM distribution performed in Section 2.3. The qf of the NTM-G family is discussed in detail in the following proposition.

Proposition 4. The of of the NTM-G family is

$$
Q_{N T M-G}(u)=Q_{G}\left(\frac{1}{\alpha} \log \left[-\alpha W\left(\frac{u d_{\alpha}-1}{\alpha e^{1 / \alpha}}\right)\right]\right), \quad u \in(0,1),
$$

where $Q_{G}(x)$ denotes the af associated to $G(x)$.

Proof. The proof is a consequence of the facts that $F_{N T M-G}(x)=F_{N T M}(G(x)), x \in \mathbb{R}$, and the application of Proposition 1.

Based on this qf, the comments formulated on the qf for the NTM distribution in Section 2.3 can be transposed.

For the Weibull distribution, we have $Q_{W}(x)=c[-\log (1-x)]^{1 / k}$, implying that the qf of the NTMW distribution is

$$
Q_{N T M W}(u)=c\left[-\log \left(1-\frac{1}{\alpha} \log \left[-\alpha W\left(\frac{u d_{\alpha}-1}{\alpha e^{1 / \alpha}}\right)\right]\right)\right]^{1 / k}, \quad u \in(0,1) .
$$

This qf can be used for further quantile analysis of the NTMW distribution, beyond the scope of this paper.

3.4. Moment Analysis. The moment analysis of the NTM-G family is now being considered. Two approaches are proposed: an integral approach and a series decomposition approach.

3.4.1. Integral Approach. The following result expresses an integral representation of the ordinary moments of the NTM-G family.

Proposition 5. Let $s$ be a positive integer and $X$ be a random variable with the pdf of the NTM-G family. Then, the sth ordinary moment of $X$ can be expressed as

$$
m_{G}(s)=C_{\alpha} \alpha e^{1 / \alpha} \int_{1 / \alpha}^{e^{\alpha} / \alpha}\left[Q_{G}\left(\frac{1}{\alpha} \log (\alpha z)\right)\right]^{s}(z-1) e^{-z} \mathrm{~d} z,
$$

provided that it exists.

Proof. We proceed as the proof of Proposition 2. By making the incremental changes of variable $y=G(x)$, then $z=e^{\alpha y} / \alpha$, and we have

$$
\begin{aligned}
m_{G}(s) & =\int_{-\infty}^{+\infty} x^{s} f_{N T M-G}(x) \mathrm{d} x \\
& =C_{\alpha} \int_{-\infty}^{+\infty} x^{s} g(x)\left(e^{\alpha G(x)}-\alpha\right) \exp \left[\alpha G(x)-\frac{1}{\alpha}\left(e^{\alpha G(x)}-1\right)\right] \mathrm{d} x \\
& =C_{\alpha} \int_{0}^{1}\left[Q_{G}(y)\right]^{s}\left(e^{\alpha y}-\alpha\right) \exp \left[\alpha y-\frac{1}{\alpha}\left(e^{\alpha y}-1\right)\right] \mathrm{d} y \\
& =C_{\alpha} \alpha e^{1 / \alpha} \int_{1 / \alpha}^{e^{\alpha} / \alpha}\left[Q_{G}\left(\frac{1}{\alpha} \log (\alpha z)\right)\right]^{s}(z-1) e^{-z} \mathrm{~d} z .
\end{aligned}
$$

The demonstration of the proposition is now complete. 

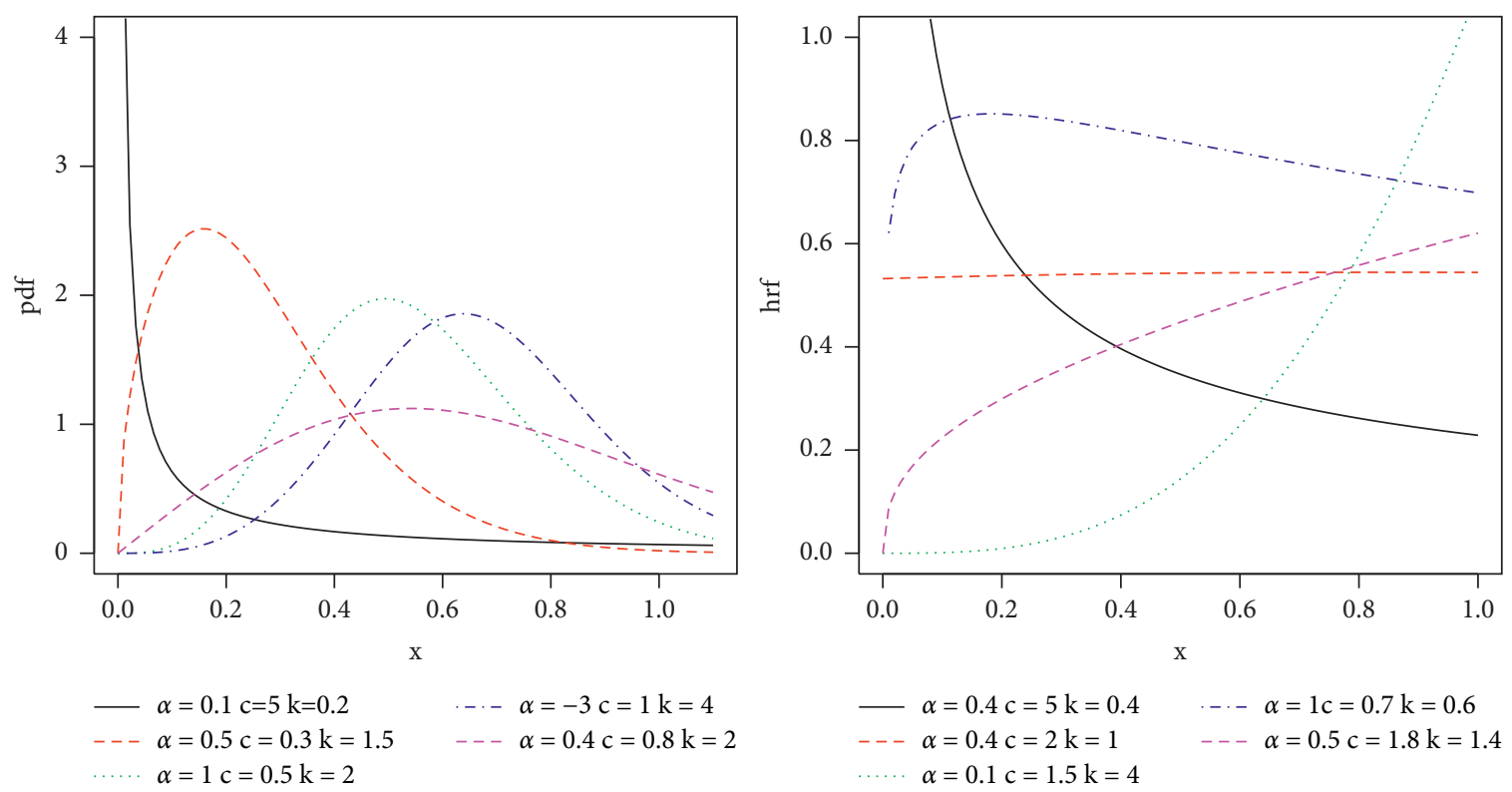

Figure 1: Plots showing different shapes of the pdf (a) and hrf (b) of the NTMW distribution.

According to the complexity of $Q_{G}(x)$, the integral in Proposition 5 may have further development. Based on ordinary moments, the well-known moment measures as presented in Section 2.4 can be defined in a similar way; mean $m_{G}(1)$, standard deviation $\sigma$, moments skewness coefficient $S$, and moment kurtosis coefficient $K$, among others.

In the setting of the NTMW distribution, we have

$$
m_{G}(s)=C_{\alpha} \alpha e^{1 / \alpha} c^{s} \int_{1 / \alpha}^{e^{\alpha} / \alpha}\left[-\log \left(1-\frac{1}{\alpha} \log (\alpha z)\right)\right]^{s / k}(z-1) e^{-z} \mathrm{~d} z .
$$

This integral seems to be not reported in the literature. It can, however, be computed by using mathematical software.

3.4.2. Series Approach. The following result proposes another approach for the calculation of ordinary moments in the form of a series expansion. This may be of interest for indepth mathematical or computational manipulations.
Proposition 6. Let $s$ be a positive integer and $X$ be a random variable with the pdf of the NTM-G family. Under the condition that sums and integral can be inverted, the sth ordinary moment of $X$ can be expanded as

$$
m_{G}(s)=\sum_{k, \ell=0}^{+\infty} A_{k, \ell} T_{\ell ; G}(s),
$$

where $A_{k, \ell}=C_{\alpha} e^{1 / \alpha}(-1)^{k} \alpha^{\ell-k}\left((k+2)^{\ell}-\alpha(k+1)^{\ell}\right) /(k ! \ell !)$ and

$$
T_{\ell ; G}(s)=\int_{-\infty}^{+\infty} x^{s} g(x) G(x)^{\ell} \mathrm{d} x
$$

Proof. First, we recall that $m_{G}(s)=\int_{-\infty}^{+\infty} x^{s} f_{N T M-G}(x) \mathrm{d} x$. We can apply the Taylor series to the exponential function several times after rewriting $f_{N T M-G}(x)$ in a suitable manner, yielding the following expansions in turn:

$$
\begin{aligned}
f_{N T M-G}(x) & =C_{\alpha} e^{1 / \alpha} g(x)\left(e^{2 \alpha G(x)}-\alpha e^{\alpha G(x)}\right) \exp \left[-\frac{1}{\alpha} e^{\alpha G(x)}\right] \\
& =C_{\alpha} e^{1 / \alpha} g(x)\left(e^{2 \alpha G(x)}-\alpha e^{\alpha G(x)}\right) \sum_{k=0}^{+\infty} \frac{(-1)^{k}}{\alpha^{k} k !} e^{\alpha k G(x)} \\
& =C_{\alpha} e^{1 / \alpha} g(x)\left[\sum_{k=0}^{+\infty} \frac{(-1)^{k}}{\alpha^{k} k !} e^{\alpha(k+2) G(x)}-\alpha \sum_{k=0}^{+\infty} \frac{(-1)^{k}}{\alpha^{k} k !} e^{\alpha(k+1) G(x)}\right]
\end{aligned}
$$




$$
\begin{aligned}
& =C_{\alpha} e^{1 / \alpha} g(x)\left[\sum_{k=0}^{+\infty} \frac{(-1)^{k}}{\alpha^{k} k !} \sum_{\ell=0}^{+\infty} \frac{\alpha^{\ell}(k+2)^{\ell}}{\ell !} G(x)^{\ell}-\alpha \sum_{k=0}^{+\infty} \frac{(-1)^{k}}{\alpha^{k} k !} \sum_{\ell=0}^{+\infty} \frac{\alpha^{\ell}(k+1)^{\ell}}{\ell !} G(x)^{\ell}\right] \\
& =\sum_{k, \ell=0}^{+\infty} A_{k, \ell}\left\{g(x) G(x)^{\ell}\right\} .
\end{aligned}
$$

The sth ordinary moment of $X$ is expanded under the condition that sums and integrals can be inverted, which is primarily determined by the definition of $G(x)$. In this case, we have

$$
m_{G}(s)=\sum_{k, \ell=0}^{+\infty} A_{k, \ell} \int_{-\infty}^{+\infty} x^{s} g(x) G(x)^{\ell} \mathrm{d} x=\sum_{k, \ell=0}^{+\infty} A_{k, \ell} T_{\ell ; G}(s) .
$$

The stated result is obtained.

The integral term $T_{\ell ; G}(s)$ can be expressed in the context of the NTMW distribution, and the series expansion becomes explicit. In addition, by taking an integer $K$ large enough, we can approximate $m_{G}(s)$ as $m_{G}(s) \approx \sum_{k, \ell=0}^{K} A_{k, \ell} T_{\ell ; G}(s)$.

3.5. Numerical Study. We now provide a numerical illustration of the moment analysis. In this regard, values of moment measures of the NTMW distribution are calculated for selected values of the parameters. These values are presented in Tables 2-4.

We can see from Tables 2-4 that, for the considered values, when $\alpha$ increases, $m$ (1) tends to increase, but $S$ and $K$ tend to decrease. Additionally, when $k$ increases, $S$ and $K$ tend to decrease.

\section{Applied Study}

In this section, we use the ML procedure to suggest some estimates for the unknown parameters of the NTMW model. Simulation experiments are used to evaluate the efficiency of these estimates. Then, two real-life datasets are used to suit the proposed distribution, with comparisons to valuable competitors.

4.1. ML Procedure. Let us now describe the ML procedure employed to estimate the NTMW model parameters. We denote by $x_{1}, \ldots, x_{n}$ some generic data that are supposed to be observed independently from a random variable with a distribution belonging to the NTM-G family. With this data, the total likelihood function is defined by $\mathscr{L}(\alpha, \zeta)=\prod_{i=1}^{n} f_{N T M-G}\left(x_{i}\right)$, where $\zeta$ denotes the possible parameters involved in $G(x)$ and thus in $g(x)$. In an expanded form, the total likelihood function becomes

$$
\begin{aligned}
\mathscr{L}(\alpha, \zeta)= & C_{\alpha}^{n}\left[\prod_{i=1}^{n} g\left(x_{i}\right)\right]\left[\prod_{i=1}^{n}\left(e^{\alpha G\left(x_{i}\right)}-\alpha\right)\right] \exp \\
& {\left[\alpha \sum_{i=1}^{n} G\left(x_{i}\right)-\frac{1}{\alpha} \sum_{i=1}^{n}\left(e^{\alpha G\left(x_{i}\right)}-1\right)\right] . }
\end{aligned}
$$

The total log-likelihood function is derived as $\ell(\alpha, \zeta)=\log [\mathscr{L}(\alpha, \zeta)]$, that is, in an expanded form,

$$
\begin{aligned}
\ell(\alpha, \zeta)= & n \log \left(C_{\alpha}\right)+\sum_{i=1}^{n} \log \left[g\left(x_{i}\right)\right]+\sum_{i=1}^{n} \log \left(e^{\alpha G\left(x_{i}\right)}-\alpha\right) \\
& +\alpha \sum_{i=1}^{n} G\left(x_{i}\right)-\frac{1}{\alpha} \sum_{i=1}^{n}\left(e^{\alpha G\left(x_{i}\right)}-1\right) .
\end{aligned}
$$

The ML estimates (MLEs) of $(\alpha, \zeta)$ are defined as $(\widehat{\alpha}, \widehat{\zeta})=$ $\operatorname{argmax}_{(\alpha, \zeta)} \mathscr{L}(\alpha, \zeta)$ or equivalently $(\widehat{\alpha}, \widehat{\zeta})=\operatorname{argmax}_{(\alpha, \zeta)} \ell$ $(\alpha, \zeta)$. Thus, when $\ell(\alpha, \zeta)$ is differentiable, $(\widehat{\alpha}, \zeta)$ can be obtained by solving the following equations simultaneously according to the parameters: $\partial \ell(\alpha, \zeta) / \partial \alpha=0$ and $\partial \ell(\alpha, \zeta) / \partial \zeta=0$, where

$$
\begin{aligned}
\frac{\partial \ell(\alpha, \zeta)}{\partial \alpha}= & n \frac{\partial C_{\alpha} / \partial \alpha}{C_{\alpha}}+\sum_{i=1}^{n} \frac{G\left(x_{i}\right) e^{\alpha G\left(x_{i}\right)}-1}{e^{\alpha G\left(x_{i}\right)}-\alpha} \\
& +\sum_{i=1}^{n} G\left(x_{i}\right)\left[1-\frac{1}{\alpha} e^{\alpha G\left(x_{i}\right)}\right]+\frac{1}{\alpha^{2}} \sum_{i=1}^{n}\left(e^{\alpha G\left(x_{i}\right)}-1\right),
\end{aligned}
$$

with

$$
\begin{aligned}
\frac{\partial C_{\alpha}}{\partial \alpha}= & \exp \left[\alpha-\frac{1}{\alpha}\left(e^{\alpha}-1\right)\right] \frac{\left(e^{\alpha}-1\right) / \alpha^{2}-e^{\alpha} / \alpha+1}{\left\{1-\exp \left[\alpha-\left(e^{\alpha}-1\right) / \alpha\right]\right\}^{2}}, \\
\frac{\partial \ell(\alpha, \zeta)}{\partial \zeta}= & \sum_{i=1}^{n} \frac{\partial g\left(x_{i}\right) / \partial \zeta}{g\left(x_{i}\right)}+\alpha \sum_{i=1}^{n} \frac{e^{\alpha G\left(x_{i}\right)} \partial G\left(x_{i}\right) / \partial \zeta}{e^{\alpha G\left(x_{i}\right)}-\alpha} \\
& +\sum_{i=1}^{n} \frac{\partial G\left(x_{i}\right)}{\partial \zeta}\left(\alpha-e^{\alpha G\left(x_{i}\right)}\right) .
\end{aligned}
$$

Due to the complex nature of these equations, there are few chances to obtain closed-form expressions for $(\widehat{\alpha}, \widehat{\zeta})$. Numerical techniques such as the (quasi) Newton-Raphson method can be used to find precise numerical solutions. An advantage of using the ML procedure is that, under wellidentified conditions, the underlying asymptotic distribution of $(\widehat{\alpha}, \widehat{\zeta})$ can be approximated by a multivariate normal distribution with a mean vector as $(\alpha, \zeta)$ and the following matrix of covariance: $\left.\left[-\partial^{2} \ell(\alpha, \zeta) / \partial \xi \partial \xi^{t}\right]^{-1}\right|_{(\alpha, \zeta)=(\widehat{\alpha}, \widehat{\zeta})}$ with $\xi=(\alpha, \zeta)$. This matrix can be computed with the help of mathematical software. Thanks to this standard multivariate distribution, we can construct approximate confidence intervals (CIs) for the model parameters at a certain level, say 
TABLE 2: Values of moment measures of the NTMW distribution for $c=0.5$ and $k=0.5$ and different values for $\alpha$.

\begin{tabular}{lccccccc}
\hline$\alpha$ & $m_{G}(1)$ & $m_{G}(2)$ & $m_{G}(3)$ & $m_{G}(4)$ & $\sigma^{2}$ & $S$ \\
\hline-0.9 & 0.446 & 2.207 & 31.722 & 879.446 & 2.008 & 10.173 \\
-0.7 & 0.48 & 2.413 & 34.789 & 965.123 & 2.183 & 9.781 \\
-0.5 & 0.519 & 2.656 & 38.418 & 1066.546 & 2.386 & 9.377 \\
-0.3 & 0.567 & 2.959 & 42.966 & 1193.818 & 2.637 & 8.944 \\
-0.1 & 0.629 & 3.358 & 48.992 & 1362.664 & 2.962 & 8.465 \\
0.1 & 0.712 & 3.905 & 57.299 & 1595.782 & 3.398 & 7.932 \\
0.3 & 0.824 & 4.666 & 68.931 & 1922.617 & 3.987 & 7.35 \\
0.5 & 0.977 & 5.716 & 85.021 & 2374.997 & 4.762 & 174.024 \\
0.7 & 1.177 & 7.108 & 106.368 & 2975.182 & 5.722 & 6.749 \\
0.9 & 1.426 & 8.83 & 132.714 & 3715.149 & 6.798 & 6.176 \\
\hline
\end{tabular}

TABLE 3: Values of moment measures of the NTMW distribution for $c=0.5$ and $k=1.2$ and different values for $\alpha$.

\begin{tabular}{lccccccc}
\hline$\alpha$ & $m_{G}(1)$ & $m_{G}(2)$ & $m_{G}(3)$ & $m_{G}(4)$ & $\sigma^{2}$ & $S$ \\
\hline-0.9 & 0.296 & 0.18 & 0.171 & 0.221 & 0.093 & 2.245 \\
-0.7 & 0.308 & 0.193 & 0.186 & 0.241 & 0.097 & 2.174 \\
-0.5 & 0.323 & 0.207 & 0.202 & 0.264 & 0.103 & 2.099 \\
-0.3 & 0.339 & 0.224 & 0.223 & 0.293 & 0.109 & 2.015 \\
-0.1 & 0.36 & 0.247 & 0.249 & 0.331 & 0.117 & 1.919 \\
0.1 & 0.387 & 0.276 & 0.285 & 0.383 & 0.126 & 8.922 \\
0.3 & 0.423 & 0.316 & 0.335 & 0.455 & 0.137 & 8.886 \\
0.5 & 0.47 & 0.37 & 0.402 & 0.555 & 0.149 & 1.678 \\
0.7 & 0.53 & 0.44 & 0.491 & 0.686 & 0.16 & 1.536 \\
0.9 & 0.603 & 0.527 & 0.602 & 0.849 & 0.164 & 1.399 \\
\hline
\end{tabular}

TABLE 4: Values of moment measures of the NTMW distribution for $c=0.5$ and $k=2$ and different values for $\alpha$.

\begin{tabular}{lccccccc}
\hline$\alpha$ & $m_{G}(1)$ & $m_{G}(2)$ & $m_{G}(3)$ & $m_{G}(4)$ & $\sigma^{2}$ & $S$ & $K$ \\
\hline-0.9 & 0.327 & 0.147 & 0.083 & 0.056 & 0.04 & 1.084 & 4.438 \\
-0.7 & 0.336 & 0.154 & 0.088 & 0.06 & 0.042 & 1.044 & 4.3 \\
-0.5 & 0.346 & 0.162 & 0.095 & 0.065 & 0.043 & 1 & 4.156 \\
-0.3 & 0.357 & 0.172 & 0.102 & 0.071 & 0.044 & 0.95 & 4.003 \\
-0.1 & 0.372 & 0.184 & 0.112 & 0.079 & 0.046 & 0.891 & 3.837 \\
0.1 & 0.39 & 0.2 & 0.124 & 0.089 & 0.048 & 0.82 & 3.656 \\
0.3 & 0.414 & 0.221 & 0.141 & 0.103 & 0.05 & 0.733 & 3.471 \\
0.5 & 0.444 & 0.249 & 0.164 & 0.122 & 0.052 & 0.633 & 3.311 \\
0.7 & 0.483 & 0.285 & 0.194 & 0.147 & 0.052 & 0.535 & 3.236 \\
0.9 & 0.53 & 0.329 & 0.23 & 0.178 & 0.048 & 0.505 & 3.317 \\
\hline
\end{tabular}

$100 p \%$ with $p \in(0,1)$; the formula for the associated lower bounds (LBs) and upper bounds (UBs) remains quite standard. All technical details and results of the ML procedure can be found in [27].

4.2. Simulation Work. The methodology of the ML procedure presented above can be applied to the NTMW distribution by taking $G(x)=G_{W}(x)$ and $g(x)=g_{W}(x)$. In this case, $\xi=(\alpha, c, k)$ becomes the unknown vector of parameters. Thus, it is natural to think that the MLEs are adequate at estimating the parameters $\alpha, c$, and $k$. We illustrate this claim by providing a simulation study to assess the behavior of these MLEs by considering the mean square errors (MSEs), lower bounds (LBs) of CIs, upper bounds (UBs) of CIs, and the corresponding average lengths (ALs) with the levels chosen as $90 \%$ or $95 \%$.
The results are obtained using the $R$ software (see [28]). Our simulation process is designed as follows :

(i) As a first operation, 5000 samples of values with sizes $n=100,200,300$, and 1000 are generated from the NTMW distribution

(ii) Values of true parameters $\alpha, c$, and $k$, in this order, are taken as Set1 $(-0.5,0.5,0.7)$, Set2 $(-0.3,0.5,0.8), \quad$ Set3 $(0.5,0.5,1.5), \quad$ and Set4 $(0.3,0.8,1.2)$

(iii) The MLEs, MSEs, LBs, UBs, and ALs of the CIs for the selected values of the parameters are calculated

(iv) The numerical results are given in Tables 5-8

The values in Tables 5-8 for the four considered sets of parameters indicate that when $n$ increases, the MLEs are near equal to the expected values. Also, the MSEs and ALs decrease and tend to 0 as $n$ becomes consequent. Overall, the simulation work found that estimating the model parameters using the ML procedure is appropriate.

4.3. Application to Real Datasets. In this section, the fits of the NTMW model are compared to those of modified Weibull models. Precisely, we consider the beta Weibull (BW) model by Famoye et al. [29], $M$ Weibull (MW) model by Almarashi and Elgarhy [7], exponentiated Weibull (EW) model by Mudholkar and Srivastava [30], and classical $M$ model. The cdf related to these models is described below.

(i) The underlying cdf of the BW model is given by 
TAble 5: Values of the MLEs, MSEs, and elements of CIs associated with the NTMW model parameters for Set1.

\begin{tabular}{|c|c|c|c|c|c|c|c|c|}
\hline \multirow{2}{*}{$n$} & \multirow{2}{*}{ MLE } & \multirow{2}{*}{ MSE } & \multicolumn{3}{|c|}{$90 \%$} & \multicolumn{3}{|c|}{$95 \%$} \\
\hline & & & LB & UB & $\mathrm{AL}$ & LB & UB & $\mathrm{AL}$ \\
\hline \multirow{3}{*}{100} & -0.4548 & 0.0167 & -0.7616 & -0.2080 & 0.5536 & -0.8146 & -0.1550 & 0.6596 \\
\hline & 0.8435 & 1.5680 & 0.5743 & 2.6614 & 3.2358 & -0.8842 & 2.9712 & 3.8554 \\
\hline & 0.7862 & 0.2260 & -0.4909 & 2.0634 & 2.5543 & -0.7355 & 2.3079 & 3.0434 \\
\hline \multirow{3}{*}{200} & -0.4685 & 0.0150 & -0.6661 & -0.2708 & 0.3953 & -0.7040 & -0.2330 & 0.4710 \\
\hline & 0.8130 & 0.3653 & -0.1579 & 1.7839 & 1.9417 & -0.3438 & 1.9698 & 2.3136 \\
\hline & 0.7757 & 0.1368 & -0.1556 & 1.6271 & 1.7827 & -0.3263 & 1.7978 & 2.1241 \\
\hline \multirow{3}{*}{300} & -0.4872 & 0.0037 & -0.6669 & -0.3074 & 0.3595 & -0.7014 & -0.2730 & 0.4283 \\
\hline & 0.6564 & 0.1103 & -0.0891 & 1.4019 & 1.4910 & -0.2318 & 1.5447 & 1.7765 \\
\hline & 0.7454 & 0.0883 & -0.0394 & 1.5302 & 1.5695 & -0.1896 & 1.6804 & 1.8701 \\
\hline \multirow{3}{*}{1000} & -0.4913 & 0.0018 & -0.5919 & -0.3907 & 0.2012 & -0.6112 & -0.3714 & 0.2397 \\
\hline & 0.5616 & 0.0373 & 0.2087 & 0.9145 & 0.7059 & 0.1411 & 0.9821 & 0.8410 \\
\hline & 0.6869 & 0.0396 & 0.3311 & 1.2426 & 0.9115 & 0.2438 & 1.3299 & 1.0861 \\
\hline
\end{tabular}

TABle 6: Values of the MLEs, MSEs, and elements of CIs associated with the NTMW model parameters for Set2.

\begin{tabular}{|c|c|c|c|c|c|c|c|c|}
\hline \multirow{2}{*}{$n$} & \multirow{2}{*}{ MLE } & \multirow{2}{*}{ MSE } & \multicolumn{3}{|c|}{$90 \%$} & \multicolumn{3}{|c|}{$95 \%$} \\
\hline & & & LB & UB & $\mathrm{AL}$ & LB & UB & $\mathrm{AL}$ \\
\hline \multirow{3}{*}{100} & -0.3208 & 0.0043 & -0.5306 & -0.0911 & 0.4396 & -0.5727 & -0.0490 & 0.5237 \\
\hline & 0.6541 & 0.5367 & -0.5498 & 1.8579 & 2.4077 & -0.7803 & 2.0884 & 2.8687 \\
\hline & 1.0716 & 0.9261 & -0.9936 & 3.1369 & 4.1305 & -1.3891 & 3.5324 & 4.9215 \\
\hline \multirow{3}{*}{200} & -0.3119 & 0.0038 & -0.4477 & -0.1762 & 0.2715 & -0.4737 & -0.1502 & 0.3235 \\
\hline & 0.6250 & 0.3725 & -0.2091 & 1.4592 & 1.6683 & -0.3689 & 1.6189 & 1.9878 \\
\hline & 0.9152 & 0.6074 & -0.3725 & 2.6030 & 2.9755 & -0.6574 & 2.8878 & 3.5452 \\
\hline \multirow{3}{*}{300} & -0.3140 & 0.0031 & -0.4164 & -0.1916 & 0.2248 & -0.4380 & -0.1701 & 0.2679 \\
\hline & 0.5621 & 0.1282 & -0.0488 & 1.1731 & 1.2219 & -0.1658 & 1.2901 & 1.4559 \\
\hline & 0.8774 & 0.3450 & -0.1100 & 2.0648 & 2.1748 & -0.3182 & 2.2730 & 2.5912 \\
\hline \multirow{3}{*}{1000} & -0.3039 & 0.0008 & -0.3690 & -0.2387 & 0.1303 & -0.3815 & -0.2263 & 0.1553 \\
\hline & 0.5229 & 0.0214 & 0.2001 & 0.8458 & 0.6456 & 0.1383 & 0.9076 & 0.7693 \\
\hline & 0.7943 & 0.0613 & 0.3152 & 1.2733 & 0.9581 & 0.2234 & 1.3651 & 1.1416 \\
\hline
\end{tabular}

TABLe 7: Values of the MLEs, MSEs, and elements of CIs associated with the NTMW model parameters for Set3.

\begin{tabular}{|c|c|c|c|c|c|c|c|c|}
\hline \multirow{2}{*}{$n$} & \multirow{2}{*}{ MLE } & \multirow{2}{*}{ MSE } & \multicolumn{3}{|c|}{$90 \%$} & \multicolumn{3}{|c|}{$95 \%$} \\
\hline & & & LB & UB & $\mathrm{AL}$ & LB & UB & $\mathrm{AL}$ \\
\hline \multirow{3}{*}{100} & 0.6612 & 0.1665 & 0.1043 & 1.2181 & 1.1138 & -0.0023 & 1.3248 & 1.3270 \\
\hline & 0.7871 & 0.4728 & -0.2513 & 1.8256 & 2.0768 & -0.4501 & 2.0244 & 2.4745 \\
\hline & 1.4427 & 0.0994 & 1.0430 & 1.8424 & 0.7994 & 0.9665 & 1.9190 & 0.9525 \\
\hline \multirow{3}{*}{200} & 0.4777 & 0.0097 & 0.2749 & 0.6805 & 0.4056 & 0.2360 & 0.7193 & 0.4833 \\
\hline & 0.4561 & 0.0408 & 0.0964 & 0.8158 & 0.7194 & 0.0275 & 0.8847 & 0.8572 \\
\hline & 1.4844 & 0.0070 & 1.1985 & 1.7703 & 0.5717 & 1.1438 & 1.8250 & 0.6812 \\
\hline \multirow{3}{*}{300} & 0.4941 & 0.0046 & 0.3233 & 0.6648 & 0.3415 & 0.2906 & 0.6975 & 0.4069 \\
\hline & 0.5217 & 0.0164 & 0.1963 & 0.8471 & 0.6507 & 0.1340 & 0.9094 & 0.7754 \\
\hline & 1.4864 & 0.0064 & 1.2550 & 1.7179 & 0.4630 & 1.2106 & 1.7623 & 0.5516 \\
\hline \multirow{3}{*}{1000} & 0.5000 & 0.0012 & 0.4066 & 0.5934 & 0.1868 & 0.3887 & 0.6112 & 0.2225 \\
\hline & 0.4861 & 0.0040 & 0.3198 & 0.6523 & 0.3325 & 0.2880 & 0.6841 & 0.3962 \\
\hline & 1.5160 & 0.0053 & 1.3887 & 1.6432 & 0.2545 & 1.3644 & 1.6676 & 0.3032 \\
\hline
\end{tabular}

$$
F_{B W}(x)=\frac{1}{B(\alpha, \beta)} \int_{0}^{1-e^{-(x / \lambda)^{\theta}}} t^{\alpha-1}(1-t)^{\beta-1} \mathrm{~d} t, \quad x>0
$$

and $F_{B W}(x)=0$ for $x \leq 0$, with $\alpha, \beta, \lambda$, and $\theta>0$, where $B(\alpha, \beta)$ denotes the standard beta function, i.e., $B(\alpha, \beta)=\int_{0}^{1} t^{\alpha-1}(1-t)^{\beta-1} \mathrm{~d} t$

(ii) The underlying cdf of the MW model is given by 
TABLE 8: Values of the MLEs, MSEs, and elements of CIs associated with the NTMW model parameters for Set4.

\begin{tabular}{|c|c|c|c|c|c|c|c|c|}
\hline \multirow{2}{*}{$n$} & \multirow{2}{*}{ MLE } & \multirow{2}{*}{ MSE } & \multicolumn{3}{|c|}{$90 \%$} & \multicolumn{3}{|c|}{$95 \%$} \\
\hline & & & LB & UB & $\mathrm{AL}$ & LB & UB & $\mathrm{AL}$ \\
\hline \multirow{3}{*}{100} & 0.3214 & 0.0189 & 0.1339 & 0.4688 & 0.3349 & 0.1018 & 0.5009 & 0.3990 \\
\hline & 0.8798 & 0.5527 & 0.1141 & 1.6456 & 1.5315 & -0.0326 & 1.7922 & 1.8248 \\
\hline & 1.4424 & 0.2932 & 0.9472 & 1.9376 & 0.9904 & 0.8524 & 2.0324 & 1.1801 \\
\hline \multirow{3}{*}{200} & 0.3111 & 0.0033 & 0.1931 & 0.4291 & 0.2359 & 0.1705 & 0.4516 & 0.2811 \\
\hline & 0.8511 & 0.0925 & 0.3345 & 1.4476 & 1.1131 & 0.2279 & 1.5542 & 1.3263 \\
\hline & 1.2647 & 0.0336 & 0.9498 & 1.4595 & 0.5096 & 0.9010 & 1.5083 & 0.6072 \\
\hline \multirow{3}{*}{300} & 0.2979 & 0.0029 & 0.2086 & 0.3872 & 0.1786 & 0.1915 & 0.4043 & 0.2128 \\
\hline & 0.8195 & 0.0736 & 0.4051 & 1.2340 & 0.8289 & 0.3257 & 1.3133 & 0.9876 \\
\hline & 1.2368 & 0.0299 & 1.0237 & 1.4499 & 0.4263 & 0.9829 & 1.4907 & 0.5079 \\
\hline \multirow{3}{*}{1000} & 0.3064 & 0.0008 & 0.2550 & 0.3579 & 0.1029 & 0.2451 & 0.3677 & 0.1226 \\
\hline & 0.8058 & 0.0161 & 0.6070 & 1.0846 & 0.4776 & 0.5613 & 1.1303 & 0.5690 \\
\hline & 1.2061 & 0.0041 & 1.0914 & 1.3207 & 0.2293 & 1.0695 & 1.3426 & 0.2732 \\
\hline
\end{tabular}

TABLE 9: Values of the estimates for dataset I.

\begin{tabular}{lccc}
\hline Model & \multicolumn{2}{c}{ MLEs (SEs in parentheses) } \\
\hline NTMW & 0.7585 & 2.8517 & 2.9959 \\
$(\alpha, c, k)$ & $(0.2256)$ & $(0.2383)$ & $(0.5106)$ \\
BW & 0.7536 & 0.1509 & 0.0806 \\
$(\alpha, \beta, \lambda, \theta)$ & $(0.1689)$ & $(0.0204)$ & $(0.0018)$ \\
MW & 0.7239 & 2.7581 & 3.0387 \\
$(\alpha, \beta, \gamma)$ & $(0.5179)$ & $(0.2573)$ & $(0.5596)$ \\
EW & 0.0118 & 3.8145 & 0.8320 \\
$(\alpha, \beta, \gamma)$ & $(0.0111)$ & $(0.6202)$ & $(0.2368)$ \\
M & 0.05675 & & \\
$(\alpha)$ & $(0.0474)$ & & \\
\hline
\end{tabular}

$$
F_{M W}(x)=e^{1 / \alpha}\left(1-e^{-(x / \beta)^{\gamma}}\right)^{-\alpha} \exp \left[-\frac{1}{\alpha}\left(1-e^{-(x / \beta)^{\gamma}}\right)^{-\alpha}\right], \quad x>0,
$$

and $F_{M W}(x)=0$ for $x \leq 0$, with $\alpha, \beta$, and $\gamma>0$

(iii) The underlying cdf of the EW model is given by

$$
F_{E W}(x)=\left(1-e^{-(x / \alpha)^{\beta}}\right)^{\gamma}, \quad x>0,
$$

and $F_{E W}(x)=0$ for $x \leq 0$, with $\alpha, \beta$, and $\gamma>0$

(iv) The underlying cdf of the $M$ model is given by equation (1), with $\alpha \in(-\infty, 1) /\{0\}$

The first real dataset, called dataset I, corresponds to the breaking stress of carbon fibres (in Gba). It was researched in [31]. It contains $n=66$ data values.

The second dataset, called dataset II, was derived from studies in [8] and represents $n=84$ aircraft windshield failure times.

The $R$ software is again employed. Tables 9 and 10 show the MLEs for the parameters in each of the five models, along with their standard errors (SEs), for datasets I and II, respectively.
Classically, the estimated negative maximum log-likelihood function $(\rho)$, Akaike information criterion (AIC), Bayesian information criterion (BIC), Cramér von-Mises (W) statistic, Anderson-Darling (A) statistic, and Kolmogorov-Smirnov (KS) statistic with $p$ value ( $p$ value) are calculated in Tables 11 and 12 for datasets I and II, respectively. The model with the smallest AIC, BIC, $W, A$, and $\mathrm{KS}$, as well as the greatest $p$ value for the KS test, is the overall best model using these criteria.

Based on Tables 11 and 12, all of the models appear to be competitive in fitting the datasets, except the $M$ model, which has very small $p$ values for the KS test. The proposed NTMW model, however, outperforms the competitors on all criteria and is the best model for both datasets. Furthermore, the obtained $p$ values are all greater than 0.9 , which is quite satisfactory in terms of adequacy.

In order to visualize the efficiency of the modeling, the fitted pdfs and cdfs of each of the five models are plotted in Figures 2 and 3 , for datasets I and II, respectively.

The estimated pdfs and cdfs of the NTMW model have particularly well captured the form of the corresponding empirical objects, as shown in Figures 2 and 3. In particular, for the histograms of the datasets, the maximum and tails have received better treatment for the NTMW model than for the competitive models. 
TABLE 10: Values of the estimates for dataset II.

\begin{tabular}{lccc}
\hline Model & \multicolumn{2}{c}{ MLEs (SEs in parentheses) } \\
\hline NTMW & 0.8130 & 2.5148 & 1.9884 \\
$(\alpha, c, k)$ & $(0.1495)$ & $(0.2289)$ & $(0.2666)$ \\
BW & 0.4187 & 0.5725 & 0.0088 \\
$(\alpha, \beta, \lambda, \theta)$ & $(0.1156)$ & $(0.3904)$ & $(0.0087)$ \\
MW & -0.3072 & 2.7346 & 2.4510 \\
$(\alpha, \beta, \gamma)$ & $(0.2389)$ & $(0.1947)$ & $(0.2782)$ \\
EW & 0.0067 & 3.9254 & 0.4667 \\
$(\alpha, \beta, \gamma)$ & $(0.0052)$ & $(0.4976)$ & $(0.0940)$ \\
M & 0.0424 & & \\
$(\alpha)$ & $(0.0435)$ & & \\
\hline
\end{tabular}

TABLE 11: Values of the considered criteria for dataset I.

\begin{tabular}{lccccccc}
\hline Model & $\rho$ & AIC & BIC & W & A & KS & $p$ value \\
\hline NTMW & 85.5748 & 177.1496 & 183.7186 & 0.0802 & 0.4467 & 0.0702 & 0.9008 \\
BW & 85.3660 & 178.7321 & 187.4907 & 0.0847 & 0.4677 & 0.0805 \\
MW & 86.1691 & 178.3382 & 184.9072 & 0.0885 & 0.4648 & 0.0759 \\
EW & 85.9488 & 177.8977 & 184.4667 & 0.0867 & 0.5090 & 0.0814 & 0.8371 \\
M & 181.4929 & 364.9857 & 367.1754 & 0.1908 & 1.0226 & 0.7126 & $<0.001$ \\
\hline
\end{tabular}

TABLE 12: Values of the considered criteria for dataset II.

\begin{tabular}{lccccccc}
\hline Model & $\rho$ & AIC & BIC & W & A & KS & $p$ value \\
\hline NTMW & 127.1974 & 260.3948 & 267.7228 & 0.0478 & 0.4992 & 0.0552 \\
BW & 128.9458 & 265.8915 & 275.6621 & 0.0926 & 0.6648 & 0.0722 \\
MW & 128.2088 & 262.4177 & 269.7456 & 0.0636 & 0.4818 & 0.0810 \\
EW & 129.0980 & 264.1960 & 271.5240 & 0.0762 & 0.5916 & 0.0640 \\
M & 217.3901 & 436.7803 & 439.2229 & 0.1027 & 0.9541 & 0.6278 & 0.8769 \\
\hline
\end{tabular}
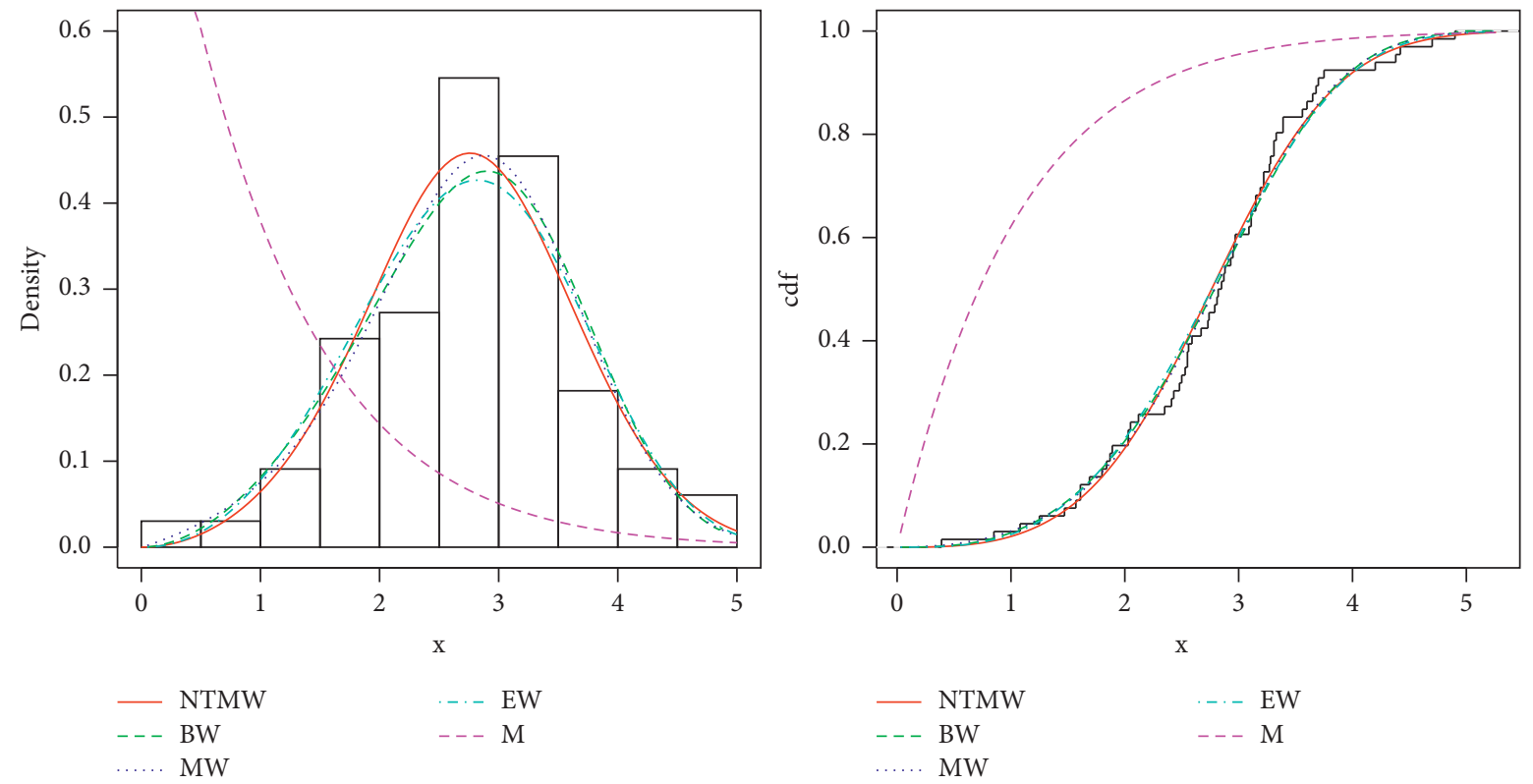

FIgURE 2: Estimated pdfs (a) and estimated cdfs (b) for the considered models for dataset I. 

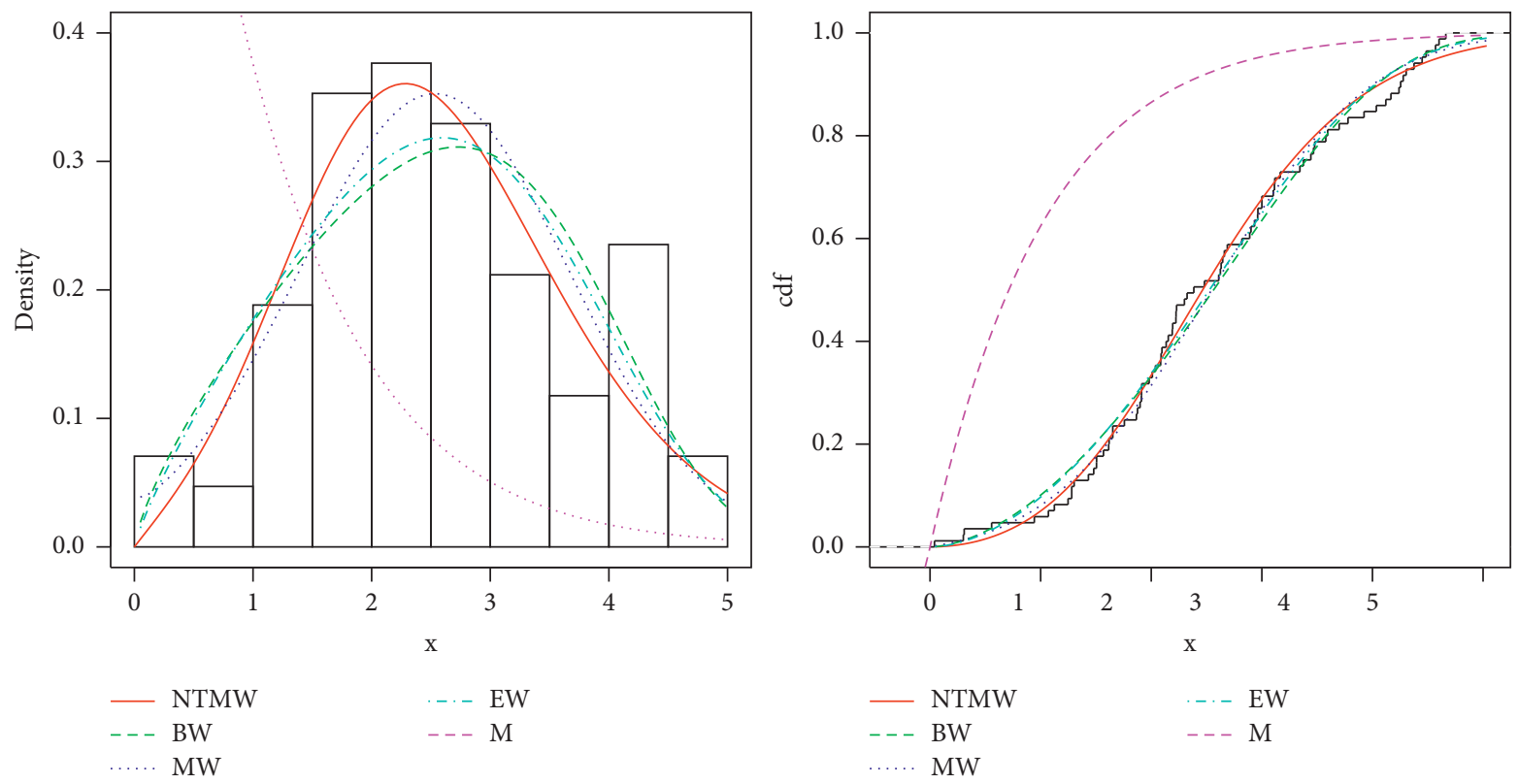

Figure 3: Estimated pdfs (a) and estimated cdfs (b) for the considered models for dataset II.

\section{Conclusion}

The Muth distribution is a versatile distribution that can serve various statistical objectives. In this study, we have briefly discussed the possibility of a unit truncated version of this distribution. Then, we exploited it to construct the new truncated Muth generated (NTM-G) family of distributions. The mathematical properties of this family have been thoroughly examined. We have outlined a special distribution of the NTM-G family, providing a motivated three-parameter modification of the Weibull distribution. It is called the new truncated Muth Weibull (NTMW) distribution. A rule-ofthumb simulation research ensures the effectiveness of the maximum likelihood procedure, which is employed to estimate the model parameters. The flexibility of the model for data fitting has been demonstrated using two realistic datasets. The NTMW model was shown to be the best for the given datasets, when compared to various competing models.

Future works of the proposed family include the applications of the distributions in important applied scenarios, such as those considered in $[32,33]$, a bivariate extended family based on the method of [34], and a discrete version following the approach of [35].

\section{Data Availability}

The data used to support the findings of the study are available from the corresponding author upon reasonable request.

\section{Conflicts of Interest}

The authors declare that they have no conflicts of interest.

\section{Acknowledgments}

This work was funded by the Deanship of Scientific Research (DSR), King Abdulaziz University, Jeddah, under Grant no.
(D-148-130-1439). The authors gratefully acknowledge the DSR technical and financial support.

\section{References}

[1] J. E. Muth, "Reliability models with positive memory derived from the mean residual life function," in The Theory and Applications of Reliability, C. P. Tsokos and I. Shimi, Eds., vol. 2, pp. 401-435, Springer, Berlin, Germany, 1977.

[2] P. Jodrá, M. D. Jimenez-Gamero, and M. V. Alba-Fernández, "On the Muth distribution," Mathematical Modelling and Analysis, vol. 20, no. 3, pp. 291-310, 2015.

[3] L. M. Leemis and J. T. McQueston, "Univariate distribution relationships," The American Statistician, vol. 62, no. 1, pp. 45-53, 2008.

[4] P. Jodrá, H. W. Gómez, M. D. Jimenez-Gamero, and M. V. Alba-Fernández, "The power Muth distribution*," Mathematical Modelling and Analysis, vol. 22, no. 2, pp. 186-201, 2017.

[5] V. K. Sharma, S. V. Singh, and K. Shekhawat, "Exponentiated Teissier distribution with increasing, decreasing and bathtub hazard functions," Journal of Applied Statistics, pp. 1-23, 2020.

[6] A. Alzaatreh, C. Lee, and F. Famoye, "A new method for generating families of continuous distributions," Metron, vol. 71, no. 1, pp. 63-79, 2013.

[7] A. M. Almarashi and M. Elgarhy, "A new Muth generated family of distributions with applications," The Journal of Nonlinear Science and Applications, vol. 11, no. 10, pp. 1171-1184, 2018.

[8] T. H. M. Abouelmagd, S. Al-mualim, M. Elgarhy, A. Z. Afify, and M. Ahmad, "Properties of the four-parameter Weibull distribution and its applications," Pakistan Journal of Statistics, vol. 33, no. 6, pp. 449-466, 2017.

[9] A. A. Al-Babtain, I. Elbatal, C. Chesneau, and F. Jamal, “The transmuted Muth generated class of distributions with applications," Symmetry, vol. 12, no. 10, p. 1677, 2020.

[10] S. V. Singh, M. Elgarhy, Z. Ahmad, V. K. Sharma, and G. G. Hamedani, Mathematical Modeling, Computational 
Intelligence Techniques and Renewable Energy: Proceedings of the First International Conference, MMCITRE 2020, Springer, Berlin, Germany, 2021.

[11] A. H. Abid and R. K. Abdulrazak, "[0, 1] truncated FréchetG generator of distributions," Applied Mathematics, vol. 7, no. 3, pp. 51-66, 2017.

[12] H. Najarzadegan, M. H. Alamatsaz, and S. Hayati, "Truncated Weibull-G more flexible and more reliable than beta-G distribution," International Journal of Statistics and Probability, vol. 6, no. 5, pp. 1-17, 2017.

[13] R. A. R. Bantan, F. Jamal, C. Chesneau, and M. Elgarhy, "Truncated inverted Kumaraswamy generated family of distributions with applications," Entropy, vol. 21, no. 11, p. 1089, 2019.

[14] M. A. Aldahlan, "Type II truncated Fréchet generated family of distributions," International Journal of Machine Intelligence and Applications, vol. 7, no. 1, pp. 221-228, 2019.

[15] M. A. Aldahlan, F. Jamal, C. Chesneau, M. Elgarhy, and I. Elbatal, "The truncated Cauchy power family of distributions with inference and applications," Entropy, vol. 22, no. 346 , pp. 1-25, 2020.

[16] A. M. Almarashi, M. Elgarhy, F. Jamal, and C. Chesneau, "The exponentiated truncated inverse Weibull generated family of distributions with applications," Symmetry, vol. 12, no. 4, pp. 1-21, 2020.

[17] F. Jamal, H. S. Bakouch, and M. A. Nasir, A truncated general$\mathrm{G}$ class of distributions with application to truncated BurrG family, RevStat, to appear, 2020.

[18] R. A. ZeinEldin, C. Chesneau, F. Jamal, M. Elgarhy, A. M. Almarashi, and S. Al-Marzouki, "Generalized truncated Fréchet generated family distributions and their applications," Computer Modeling in Engineering and Sciences, vol. 126, no. 2, pp. 791-819, 2021.

[19] A. Algarni, A. M. Almarashi, F. Jamal, C. Chesneau, and M. Elgarhy, "Truncated inverse Lomax generated family of distributions with applications to biomedical data," Journal of Medical Imaging and Health Informatics, vol. 11, pp. 24252439, 2021.

[20] R. A. R. Bantan, C. Chesneau, F. Jamal, I. Elbatal, and M. Elgarhy, "The truncated Burr X-G family of distributions: properties and applications to actuarial and financial data," Entropy, vol. 23, no. 1088, pp. 1-24, 2021.

[21] R. A. R. Bantan, C. Chesneau, F. Jamal et al., "Some new facts about the unit-Rayleigh distribution with applications," Mathematics, vol. 8, no. 11, pp. 1-23, 2020.

[22] C. Chesneau, "A note on an extreme left skewed unit distribution: theory, modelling and data fitting," Open Statistics, vol. 2, no. 1, pp. 1-23, 2021.

[23] C. Chesneau, "Study of a unit power-logarithmic distribution," Open Journal of Mathematical Sciences, vol. 5, no. 1, pp. 218-235, 2021.

[24] M. . Ç. Korkmaz, C. Chesneau, and Z. S. Korkmaz, "On the arcsecant hyperbolic normal distribution. Properties, quantile regression modeling and applications," Symmetry, vol. 13, no. 1, pp. 1-24, 2021.

[25] C. W. Topp and F. C. Leone, "A family of J-shaped frequency functions," Journal of the American Statistical Association, vol. 50, no. 269, pp. 209-219, 1955.

[26] W. Gilchrist, Statistical Modelling with Quantile Functions, CRC Press, Abingdon, UK, 2000.

[27] G. Casella and R. L. Berger, Statistical Inference, Brooks/Cole Publishing Company, Bel Air, CA, USA, 1990.
[28] R Core Team, R: A Language and Environment for Statistical Computing, R Foundation for Statistical Computing, Vienna, Austria, 2014, http://www.R-project.org/.

[29] F. Famoye, C. Lee, and O. Olumolade, "The beta-Weibull distribution," Journal of Statistical Theory and Applications, vol. 4, pp. 121-136, 2005.

[30] G. S. Mudholkar and D. K. Srivastava, "Exponentiated Weibull family for analyzing bathtub failure-rate data," IEEE Transactions on Reliability, vol. 42, no. 2, pp. 299-302, 1993.

[31] G. M. Cordeiro and A. J. Lemonte, "The-Birnbaum-Saunders distribution: an improved distribution for fatigue life modeling," Computational Statistics \& Data Analysis, vol. 55, no. 3, pp. 1445-1461, 2011.

[32] Y. Su, X. Jiang, and Z. Lin, "Simulation and relationship strength: characteristics of knowledge flows among subjects in a regional innovation system," Science Technology \& Society, p. 097172182110204, 2021.

[33] Y. Su and Y.-Q. Yu, "Spatial agglomeration of new energy industries on the performance of regional pollution control through spatial econometric analysis," The Science of the Total Environment, vol. 704, p. 135261, 2020.

[34] M. El-Morshedy and M. S. Eliwa, "Bivariate odd Weibull-G family of distributions: properties, Bayesian and non-Bayesian estimation with bootstrap confidence intervals and application," Journal of Taibah University for Science, vol. 14, pp. 331-345, 2020.

[35] M. El-Morshedy, M. S. Eliwa, and A. Tyagi, "A discrete analogue of odd Weibull-G family of distributions: properties, classical and Bayesian estimation with applications to count data," Journal of Applied Statistics, pp. 1-25, 2021. 